werden, maximal möglich sind 30 Punkte, Werte über 26 gelten als normal, der Test ist frei verfügbar (www.mocatest.org/).

\section{MoCA-Kurzform genügt meist}

Ein Team um Dr. David Roalf von der Universität in Philadelphia hat anhand einer Auswertung von 1.850 MoCA-Datensätzen geschaut, ob sich der Test bei vergleichbarer Sensitivität noch etwas straffen lässt [1]. Die Daten stammten unter anderem von rund 350 Alzheimer-Kranken, knapp 120 Patienten mit MCI, 700 mit Morbus Parkinson, 100 mit Parkinson-Demenz sowie fast 200 gesunden Älteren. Die MoCA-Werte betrugen bei den Alzheimer-Kranken im Mittel 14 Punkte, bei den übrigen lagen sie noch über 20 Punkten. Die Forscher um Roalf suchten nun die Aufgaben mit der besten Trennschärfe heraus. Insgesamt kamen sie auf acht Testfragen, mit denen sich ähnlich gute Resultate erzielen lassen wie mit der MoCA-Langform. Sie bestehen aus einem Test für das Uhrenzeichnen, einer Gedächtnisprüfung (vier Wörter werden nach einiger Zeit wiederholt), einer Rechenaufgabe mit serieller Subtraktion, der Benennung von Ort, Zeit und Datum, einer Wortfindungsübung (Nashorn auf Abbildung benennen), einem Zahlen-Buchstaben-Verbindungstest sowie einer Übung zur Wortflüssigkeit (möglichst viele Wörter in einer Minuten benennen, die mit „ $\mathrm{K}$ “ beginnen). Damit ließ sich eine MCI bei $84 \%$ der Betroffenen feststellen, eine Alzheimer-Demenz bei $96 \%$ (Spezifität: jeweils $86 \%$ und $93 \%$ ). Patienten mit Parkinson-Demenz lassen ich damit jedoch nur schwer von Parkinson-Kranken ohne Demenz abgrenzen (Sensitivität/Spezifität: $63 \% / 69 \%$ ).

\section{Große Grauzone bei Fahrtauglichkeit}

Um zu entscheiden, ob jemand noch fahrtauglich ist, können jedoch Tests wie das MoCA allenfalls grobe Anhaltspunkte liefern. Darauf deutet eine Analyse von 243 Personen, die von Ergotherapeuten und Fahrlehrern untersucht wurden [2]. Die Teilnehmer litten an den Folgen eines Schädel-Hirn-Traumas oder Schlaganfalls, hatten eine Demenz, MS oder Parkinson. 81 von ihnen waren nach dem Urteil der Experten noch fahrtauglich, 54 nicht. Bei den übrigen waren weitere Tests nötig um, die Fahrtauglichkeit festzustellen. Alle absolvierten zudem ein MoCA. Bei den Fahrtauglichen lagen die Werte bei 12 bis 30 Punkten, bei den nicht mehr fahrtauglichen zwischen 4 und 28. Bei über 27 Punkten könne man also recht sicher sein, dass die Fahrtauglichkeit nicht durch kognitive Probleme beeinträchtigt sei, bei Werten unter 12 Punkten, die den Übergang von einer leichten in eine moderate Demenz markieren, sei davon auszugehen, dass die Betroffenen nicht mehr fahren können, sagte Schulz. In dem großen Bereich zwischen 12 und 27 Punkten - also bei MCI und leichter Demenz - sei per MoCA jedoch keine Aussage zur Fahrtüchtigkeit möglich. Diese lasse nur über eine Fahrtauglichkeitsprüfung ermitteln.

Thomas Müller, Springer Medizin

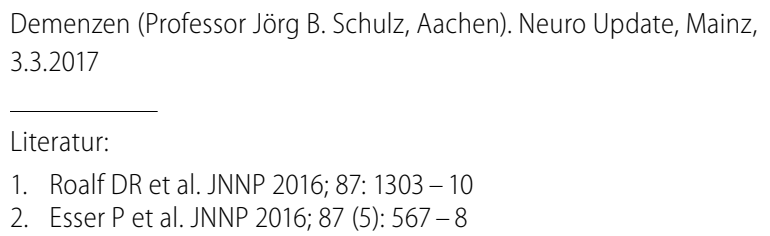

\title{
Gliomklassifikation: Genetik schlägt Histologie
}

Die neue WHO-Gliomklassifikation macht vieles komplizierter, aber auch einiges einfacher. So trennt die 1p/19q-Kodeletion klar zwischen Oligodendrogliom und Astrozytom. Die Einteilung ist vor allem für die Therapieplanung wichtig.

Im vergangenen Jahr ist die revidierte vierte Fassung der WHOKlassifikation der ZNS-Tumoren erschienen. Sie berücksichtigte sowohl klassische histopathologische Kriterien als auch molekulargenetische Eigenschaften. Bei den Gliomen habe das einerseits zu einer deutlichen Aufsplitterung, andererseits aber auch zu Vereinfachungen geführt, erläuterte Professor Uwe Schlegel, Knappschaftskrankenhaus Bochum. So wird der 1p/19q-Kodeletionsstatus zur Differenzierung zwischen Astrozytom und Oligodendrogliom herangezogen. Ein Tumor mit IDH-Mutation und $1 \mathrm{p} / 19 \mathrm{q}-$ Kodeletion ist danach ein Oligodendrogliom, fehlt die Kodeletion, liegt ein Astrogliom vor, erläuterte Schlegel. „Die Kodeletion schlägt die klassische histopathologische Zuordnung. Damit verschwinden auch das Oligoastrozytom beziehungsweise das oligoastrozytäre Mischgliom aus der Diagnostik. " Die Histopathologie bleibt jedoch weiterhin für den Tumorgrad entscheidend. Diagnosen wie „diffuses Astrozytom, nicht näher bestimmt“ oder „Oligodendrogliom, nicht näher bestimmt“ sollten nicht mehr gestellt werden. „Für uns Kliniker ist zu fordern, dass ein Gliom immer zugeordnet wird. Das ist keine akademische Spielerei, die genaue Klassifizierung erlaubt eine konkrete prognostische Einschätzung und gezielte Therapieplanung“, so Schlegel. Er nannte als Beispiel den IDH1/2-Status: Liegt eine Mutation im Gen für die Isozitratdehydrogenase (IDH) vor, haben die Patienten deutlich bessere Chancen. „Ein Glioblastom mit IDHMutation ist prognostisch günstiger einzuschätzen als ein anaplastisches Astrogliom ohne." Thomas Müller, Springer Medizin

Neuroonkologie (Prof. Uwe Schlegel, Bochum). Neuro Update, Mainz, 3.3.2017

Literatur:

1. Louis DN et al. Acta Neuropathol 2016; 131: 803

2. Van den Bent MJ et al. J Clin Oncol 2016; 34: (suppl; abstr LBA2000).

\section{Die revidierte WHO-Klassifikation...}

der Hirntumoren nutzt neben klassischen histopathologischen Kriterien auch molekulare Eigenschaften der Tumoren zur Zuordnung. In der nächsten Ausgabe von InFo Neurologie \& Psychiatrie im Mai berichtet Professor Uwe Schlegel, Bonn, in einem CME-Beitrag ausführlich über die neue Klassifikation der Gliome und deren Konsequenzen für die Therapie. 